\title{
Microbiota and Its Importance in Honey Bees
}

\author{
Ayse Ebru BORUM ${ }^{1, *}$ \\ ${ }^{1}$ Balıkesir University, Veterinary Medicine Faculty, Department of Microbiology, Balıkesir, Turkey
}

\section{Article History}

Received 14 June 2021

Accepted 05 July 2021

First Online 17 July 2021

\section{*Corresponding Author}

Tel.: +905436780123

E-mail: ebruborum@balikesir.edu.tr

\section{Keywords}

Bee

Functions

Microbial community

\begin{abstract}
In recent years, research on human and animal health has emerged as a very important microbiota and microbiome. Microbiota; has important functions in metabolism, immune system, growth and development. In recent years, it has been understood that the microbiota is effective in the protection of bee health in colony so prevent losses in honey bees. Season, flora, food sources, age of the individual, duties in the hive, chemicals used in the fight against parasites and pathogens, and many other factors can be effective on the microbiome of honey bees.
\end{abstract}

\section{Introduction}

A larger organism or microbial community living in an intensive environment is called the "microbiota". Animals that live as a community and have social relationships often use a characteristic microbiota (Pascale et al., 2018). Microbiota is defined as a mixed ecosystem of microorganisms that are critical in various metabolic functions such as regulation of glucose and lipid homeostasis, energy management and vitamin production, and regulation of satiety (Greiner \& Bäckhed, 2011; LeBlanc et al., 2013). It is effective in the production of metabolites and other substances that regulate various biochemical and physiological mechanisms. It has important functions in the functioning of the immune system and in stimulating immune responses, producing anticarcinogenic and anti-inflammatory activities_also regulates induction of protective responses of the host against pathogens and harmless antigen tolerance (Molloy, Bouladoux, \& Belkaid, 2012; Belkaid \& Harrison, 2017; Agus, Planchais, \& Sokol, 2018). The composition of the microbiota varies considerably, both between different species and within the same species. Microbiota diversity is affected by short-term changes in microbial communities and topographic differences. Specific microorganisms settle in specific environments during different growth and developmental stages of the host (Nicholson et al., 2012).

\section{Characteristics}

Insects are the most diverse animal species in terms of number of species, ecological habitats, and overall biomass. (Engel \& Moran, 2013a). Apis mellifera has a high host adaptive microbial community. The honey bee microbiota has some similarities with mammals. But in reality, it is of a simpler composition (Zheng, Steele, Leonard, Motta, \& Moran, 2018).

Young worker bees in a honeybee colony have few or no core gut microbiota (Dong et al., 2020). Microbial communities begin to colonize as they are fed orally by nurse bees (Martinson, Moy, \& Moran, 
2012; Motta, Raymann, \& Moran, 2018). In the pupal stage, bacteria in the gut microbiome are excreted together with the intestinal epithelium. The next colonization is formed by contact with other bees in the hive and by trophallaxis. (Bleau, Bouslama, Giovenazzo, \& Derome, 2020). Bacterial diversity in the gut microbiome reaches its maximum in 3-5 days after the bee matures into adulthood. Taxonomic changes occur after 3-8 days ( $\mathrm{Li}$ et al., 2017). The rectal microbiome completes its development three days after the adult stage. The ileum is more variable and its final structure appears after 8 days. Prevalence of core species, associated alteration of ileum environment and host immune response are factors affecting this process (Anderson \& Ricigliano, 2017).

Worker bees perform different tasks depending on age. New adult bees are generally responsible for hive cleaning and maintenance. Interactions between adult bees take place through social contact, cleaning and feeding behaviors. As a result, the microbiome of the species is formed (Powell, Martinson, Urban-Mead, \& Moran, 2014; Li et al., 2017). The intestinal microbiota of $A$. mellifera worker bees were investigated within 0-40 days after hatching. It was determined that Snodgrassella, Gilliamella and Frischella species were colonized in the honey bee intestine from the 1st day. Bifidobacterium, Commensalibacter, and Lactobacillus colonize within 3 days, while Gilliamella is reduced simultaneously Lactobacillus kunkeei and Bartonella sp., colonize significantly in 12 days. Shigella sp., Escherichia sp., Bacteroides sp., and Porphyromonadaceae 19 to 25 days, Commensalibacter sp. and Bifidobacterium sp. decreased at 25 days (Dong et al., 2020).

The microbiota of honey bees consists of microbial communities in different intestinal sections, also called the stomach, which is located between the esophagus and the ventriculus, which is used to store and transport nectar to the hive. Different microbial communities in the hindgut, ileum, lumen, and in the distal rectum form the honeybee microbiome (Vásquez et al., 2012; Engel \& Moran, 2013a). Parasaccharibacter sp, which is located in the microbiome, is relatively abundant in worker hypopharyngeal glands (Corby-Harris et al. 2014). Adult worker bees are thought to have about nine bacterial species in their guts (Jones et al., 2017).

Similar to humans, the microbial communities of honey bees also contain anaerobic microorganisms. The shaping of bacterial species in the microbiota occurs through social interactions between colony individuals (Alberoni, Gaggìa, Baffoni, \& Di Gioia, 2016). All bacterial species that make up the microbiota in honey bees can be grown under laboratory conditions, unlike the mammalian gut microbiome (Kwong \& Moran, 2016a). As a result of $16 \mathrm{~S}$ rDNA research, nine different bacterial species that make up $95-99.9 \%$ of the microbiota have been identified in the intestines of almost all worker bees (Jones et al., 2017; Moran,
Hansen, Powell, \& Sabree, 2012; Tola, Waweru, Hurst, Slippers, \& Paredes, 2020). Two gram negative bacterial species that are members of the Protobacteria phylum have been identified. Snodgrasella alvi, a member of the Neisseriaceae family, is a type of bacteria that does not ferment sugars and forms films directly on the intestinal wall. The other, Gilliamella apicola, lives in the central areas of the lumen and has the ability to ferment sugar. It belongs to the Orbaceae family (Jones et al., 2017; Zheng, Powell, Steele, Dietrich, \& Moran, 2017; Tola et al., 2020). Gram-positive bacteria in the Firmicutes phylum are abundant in many environments. Lactobacillus Firm-4 and Firm-5 living in the distal rectum are among these bacterial species. (Jones et al., 2017; Zheng et al., 2017). Most adult workers also have much smaller amounts of Bifidobacterium asteroides. (Kwong and Moran, 2016a; Bleau et al., 2020). These bacteria are the most important microorganisms in the honey bee gut and are called "core bacteria" (Kešnerova et al., 2020). Less dense species than Proteobacteria are; Commensalibacter sp. (Alpha 2.1) and Bartonella apis (Alpha 1); Parasaccharibacter apium, Bombella apis, Bombella mellum, Bombella favorum (Acetobacteraceae family, Alpha 2.2), Apibacter adventoris, Apibacter mensalis and Frischella perrara (Orbaceae family) (Kešnerová, Moritz, \& Engel, 2016; Kwong \& Moran, 2016b; Jones et al., 2017; Kwong, Steele, \& Moran, 2018; Bleau et al., 2020; Dong et al., 2020; Tola et al., 2020; Hilgarth, Redwitz, Ehrmann, Vogel, \& Jakob, 2021). Four Lactobacillus species, 2 Gilliamella species, 1 Bifidobacterium species and 1 Snodgrassella species, which constitute the intestinal core microbiome of honey bees, were determined. Bartonella sp. and Frischella sp. are nonnuclear members of the honeybee gut microbiome that may vary depending on the environment. Parasaccharibacter apium is a sporadic species in honey bees. This species is often isolated from both the worker's and queen's gut, and from environments such as honey and bee bread (Martinson et al., 2011; Anderson et al., 2013; Anderson, Rodrigues, Mott, Maes, \& Corby-Harris, 2016). Lactobacillus Firm-4 and Firm- 5 are actual symbionts of the gut and are rarely isolated outside of bee guts. Other Lactobacillus species, such as Lactobacillus kunkeei, can be found inside and in hive materials (Olofsson, Alsterfjord, Nilson, Butler, \& Vásquez, 2014; Milani et al., 2018; Raymann et al., 2018a).

In ileum; Lactobacillus Firm-4 and Firm-5, Snodgrasella alvi, Bifidobacterium sp. Gilliamella apicola, typical core bacteria, while Parasaccharibacter apium, Frischella perrara, Bombella apis, Bartonella apis, Apibacter advantoris and Apibacter mensalis are less common. In the honey crop part, there are fewer environmental bacteria species with Apilactobacillus kunkei predominant. The midgut microbiome is unstable. In the rectum, Bifidobacterium sp., Lactobacillus Firm-4 and Firm-5 are the dominant 
species (Subotic et al., 2019; Kešnerová et al., 2020).

Lactic acid bacteria (LAB) are an important part of the microbiom in honeybees as in other animals (Piccart, Vásquez, Piepers, De Vliegher, \& Olofsson, 2016). The microaerophilic environment of the honey bee digestive system is an ideal environment for sugars from nectar and lactic acid bacteria with a temperature of $35^{\circ} \mathrm{C}$ (lorizzo et al., 2020a). LAB plays a role in many different functions that have positive effects on the host. LAB in the microbiota prevents the colonization and invasion of the intestine by competing with the pathogens for the food in the environment (lorizzo et al., 2020b). Metabolism products such as carbon dioxide, organic acids, hydrogen peroxide or ethanol produced by the microbiota play an important role in defense against pathogens (Serna-Cock et al., 2019). $\mathrm{LAB}$ also produces bacteriocins. They can biosynthesize many different types of antagonistic molecules (Alvarez-Sieiro, Montalbán-López, Mu, D., \& Kuipers 2016). As a component of the microbiota, LAB participates in important interactions in immunomodulation (Foligne et al., 2007). LAB increases anti-inflammatory and pro-inflammatory cytokines. $L A B$ components can directly induce the immune system. It has been determined that $L A B$ also affects lipid metabolism (Kishino et al., 2013). LAB effectively protects both human and animal intestinal epithelial cells from enteric viral infections. Lactobacillus rhamnosus and Lactobacillus casei Shirota are the most effective species for protection. It has significant antiviral effects on Lactobacillus fermentum, Lactobacillus plantarum, Lactobacillus pentosus and Enterococcus faecium (Maragkoudakis, Chingwaru, Gradisnik, Tsakalidou, \& Cencic, 2010).

Thirteen bacterial species belonging to Lactobacillus and Bifidobacterium genera were determined in honey bees. Lactobacillus kunkei was found to be the most dominant species (Vásquez et al. 2012). The honey bee microbiota and bacteria isolated under hive environmental conditions are presented in Table 1.

\section{Functions}

The gut microbiome is involved in the growth, development, and reproduction of insects. It has important contributions to their metabolism. These microorganisms enhance effective digestion, promote the absorption of food and synthesize essential nutritional compounds. (Pernice, Simpson, \& Ponton, 2014). The insect gut microbiome is often limited due to the lack of interaction between individuals. However, in social insects such as honey bees, the microbiome is more various. Social interactions ensure the diversity of gut microorganisms. Thus, social insects such as honey bees have a gut microbiome that has important functions in nutrition and protection (Engel \& Moran, 2013b).

\section{Metabolic Functions}

The gut microbiota of honey bees is as important as the mammalian microbiome. Intestinal microbiota has very important functions especially in nutrient biosynthesis and biomass degradation. In nutritional function, the insect microbiome has been proven to help produce nutrients such as vitamins and amino acids that are not found in food. In biomass degradation and digestion, it was determined that the release of cellulolytic enzymes responsible for hydrolysis and the activity of microorganisms increase (Shi, Syrenne, Sun, \& Yuan, 2010; Anderson \& Ricigliano, 2017; Belkaid \& Harrison, 2017; Zheng et al., 2017).

The gut microbiota has important functions in the digestion of lipids and proteins, detoxification of secondary plant compounds. It also has positive effects on insect resistance to insecticides, while positively affecting survival, development and egg production (Jing, Qi, \& Wang, 2020).

Compared to the gut microbiota of other animals, the honeybee microbiota has effective functions in specific adaptations to a sugar-rich diet. It provides sugar uptake pathways of various phosphotransferase systems to the honeybee. Most carriers are classified in the mannose family. Nectar contains traces of mannose, but becomes highly toxic when ingested at higher concentrations. Therefore, this feature of bacteria is very important (Engel \& Moran, 2013b). The bee microbiome enriches the host with arabinose flow permeases. This family of carriers plays a role in the transport of different compounds such as sugars, antimicrobial proteins, and amino acids. Various carriers protect against various pesticides applied in agriculture (Engel \& Moran, 2013a). In addition, the intestinal microbiome plays an important role in the conversion of nectar into honey and bee secretions into propolis with its fermentation properties. It is also responsible for the freshness of honey (Pachila, Ptaszynska, Wicha, Olenska, \& Małek, 2017; Silva et al., 2017).

LAB, which constitutes an important group of bee microbiota, contributes to the nutrition of honey bees. It has been suggested that bacteria of the genus Bifidobacterium, Simonsiella and Lactobacillus can produce acetic acid, a waste product of carbohydrate metabolism. The microbiota supports the diet of bees by aiding the digestion of these compounds. After consumption, food is stored in the rectum for quite a long time. Thus, extra nutrients are obtained from rectal bacteria during wintering (Martinson et al., 2011).

This effect of the microbiota on body weight gain causes an increase in the level of vitellogenin, which is responsible for the regulation of nutrition in honey bees, by making insulin and insulin-like signal changes. This is associated with changes in gene and endocrine signal expression (Ihle, Baker, \& Amdam, 2014; Zheng 
et al., 2017). In a study, it was determined that Bifidobacterium asteroides stimulated the production of juvenile hormone and host-derived prostaglandin derivatives, which are effective in bee growth (Kešnerová et al., 2017).

Intestinal microorganisms can also prolong the lifespan of insects. It was determined that the life span of Drosophila melanogaster increased significantly after probiotic and symbiotic applications (Westfall, Lomis, \& Prakash, 2018). The microbiome synthesizes enzymes such as glycosidases and proteases. These enzymes synthesize the vitamins necessary for the host, metabolize indigestible polysaccharides, and are responsible for xenobiotic metabolism. With these features, the microbiota contributes significantly to the biochemical activities of the host -(Sommer \& Bäckhed, 2013).

Carbohydrates and oligosaccharides that cannot be digested by the host are fermented by microbiome bacteria of the genus Bifidobacterium, Bacteroides, Faecalibacterium, and Roseburia to form short-chain fatty acids (SCFAs) such as propionate, acetate, and butyrate (Jandhyala et al., 2015; Wang et al., 2020). These SCFAs formed provide rich energy sources to the host. The resulting butyrate prevents the accumulation of toxic by-products for metabolism (Jandhyala et al., 2015). Pollen is an important bee food. It is the most important source of vitamins, minerals, amino acids, and fats. Most of these are absorbed by the host midgut. Compounds that are the most difficult to digest, such as cellulose, emicellulose, pectin, remain. These compounds are degraded by the hindgut microbiota (Mollet, Leroux, Dardelle, \& Lehner, 2013).

\section{Immune System Functions}

Honeybee microbiota has important functions in regulating and stimulating the immune system of honey bees and in the formation of an effective immune response. Microbiota species are very effective in the development and morphogenesis of the immune system (Egert \& Simmering, 2016; Schroeder \& Bäckhed, 2016; Kwong, Mancenido, \& Moran, 2017). Microorganisms alter the intestinal environment, limiting the growth of insect parasites and stimulating the host immune system. Additionally, species in the microbiota produce antimicrobial peptides that play an important role in control and defense against bacterial pathogens and parasites. The overall microbiome or $S$. alvi colonization alone increases the regulation of the antimicrobial peptides apidaesin and hymenoptaesin in intestinal epithelial cells (Azambuja, Garcia, \& Ratcliffe, 2005; Kwong et al., 2017). Antimicrobial peptides are important innate immune components that play a role in the destruction of pathogenic microorganisms. A high increase in these antimicrobial peptides was detected in honey bees treated with probiotics (Kwong et al., 2017). F. perrara is a member of the microbiota in the majority of honey bees. It was determined that the host immune response increased considerably by colonizing the pylorus, which is the midgut ileum transition region (Powell et al., 2014).

The largest surface area in contact with foreign antigens originating from the external environment is the intestinal microbiota. It therefore plays a primary role in mucosal immunity (Sekirov, Russell, Antunes, \& Finlay, 2010). The microbiome controls the overgrowth and migration of microorganisms in mucosal immunity, while preventing the induction of harmful systemic immune response (Purchiaroni et al., 2013). It can affect the host by altering the composition of the gut microbiome. Ascosphaera apis, Nosema sp., Paenibacillus larvae, Melissococcus plutonius, and Serratia marcescens, such pathogens cause significant infections in honey bees. $L A B$, an important member of the microbiota, is highly effective in protecting against these pathogens. (Wu et al., 2014; Arredondo et al., 2018; lorizzi et al., 2020b; Peghaire et al., 2020).

Bacteria constituting the microbiome control intestinal homeostasis through various mechanisms including lipopolysaccharide, peptidoglycans, and flagellin. These structures interact with cell receptors in the toll like pathway. This mechanism activates intracellular signaling pathways associated with cell survival, inflammatory response, replication, and apoptosis (Evans et al., 2006; Valentini et al., 2014; Yiu, Dorweiler, \& Woo, 2017).

The gut microbiome is involved in xenobiotic metabolism. This ability ensures the preservation of the microbiome, which is a necessary condition for drugs used in the treatment of various diseases to be effective (Bäckhed et al., 2004; Vergnolle, 2016).

Honey bees are frequently exposed to a wide variety of pesticides. The microbiota also plays an important role in the detoxification of xenobiotics, particularly neonicotinoid insecticides. Microbiota microorganisms stimulate the expression of detoxification enzymes. Thus, an effective endogenous detoxification occurs in the host, increasing thiacloprid and fluvalinate resistance (Wu et al., 2020).

\section{Social and Individual Behavior Functions}

It has been proven by various studies that gut microbiota and brain compatibility. This means that gut microorganisms cause changes in host neurophysiology and insect behavior (Westfall et al., 2018; Leger \& McFrederick, 2020).

Microbiota species can alter both the volatile profiles and olfactory behaviors of the host. As a result, they regulate the way individuals make decisions regarding interacting, gathering in groups, foraging, and mating through chemical communication. In addition, intestinal microorganisms increase the host's memorization and learning capacity. Thus, it also affects the neurophysiological development of the host by supporting memory (Liberti \& Engel, 2020). It has been determined that the gut microbiome of honey 
bees influences the neurophysiology and behavior of their hosts. Levels of biogenic amines such as octopamine, dopamine, and serotonin can be altered by microbiota species, affecting host behavior. The levels of these compounds in the brain of worker bees change seasonally. Levels of the compounds increase during the summer months, when foraging activity is at its highest. The brain activity of newly developed bees, whose microbiome is not yet fully formed, is significantly lower than that of older adults (Harris \& Woodring, 1992). Microbiota plays an important role in the regulation of social behavior in honey bees (Vernier et al., 2020).

\section{Factors affecting honey bee microbiota}

Many factors affect the microbiome, such as the host species, feeding preference, host habitat, and the host life stage. Especially the high diversity of plants causes changes in the honey bee microbiome, especially Proteobacteria and Firmicutes species (Jones et al., 2017).

Many pesticides such as imidacloprid, coumaphos and chlorothalonil have serious adverse effects on bee health. It also causes significant problems by negatively affecting the structure and function of the microbiome. It also increases susceptibility to opportunistic pathogens. Honey bees are exposed to pesticides through contaminated water, nectar and pollen. It was determined that the amount of Lactobacillales decreased significantly in honey bees exposed to chlorothalonil. Non-lethal doses of insecticides such as thiamethoxam, fipronil, imidacloprid and coumaphos caused Bifidobacterium sp. and Lactobacillus sp. has been found to greatly reduce the number of (Rouzé, Moné, Delbac, Belzunces, \& Blot, 2019). The gut microbiome of honey bees exposed to glyphosate was adversely affected, affecting the total bacterial count in the gut and Bifidobacterium sp., Lactobacillus (Firm-4 and Firm-5), and decreased in S. alvi (Motta et al., 2018). Nitenpyram, a neonicotinoid insecticide, has caused significant changes in the microbiome community. Due to the insecticide effect, important metabolic changes have occurred in the host and the effectiveness of the immune system has decreased (Zhu, Qi, Xue, Niu, \& Wu, 2020). Pesticides such as imidacloprid and thiacloprid, Bifidobacterium sp., Frischella sp. and Lactobacillus (Firm-4 and Firm-5), produce numerous adverse changes in various species, causing intestinal dysbiosis (Daisley et al., 2017; Diaz, Del-Val, Ayala, \& Larsen, 2019).

Honeybee gut core microbiota is also affected by global seasonal changes (Rouzé et al., 2019). While the amount of Enterobacteriaceae decreased in autumn, Neisseriaceae increased (Bleau et al., 2020). The lowest diversity in the microbiome was detected in winter (Purchiaroni et al., 2013).

As a result of the high-fat honey bee diet containing palm oil, the amount of Gilliamella sp. in the microbiome increased significantly, while the rate of Bartonella sp. decreased (Wang et al., 2021).

Antibiotics can affect the host by causing changes in the species that form the gut microbiota. (Daisley et al., 2020). Antibiotic applications cause a decrease in bacterial species in the honeybee microbiota. This weakens the immune system of bees and increases their susceptibility to infections (Raymann, Bobay, \& Moran, 2018b). In one study, the microbiome was destroyed as a result of antibiotic administration. As a result, the expression of genes encoding antimicrobial peptides decreased and honey bees became more susceptible to Nosema ceranae infection (Li et al., 2017). In another study, disruption of the gut microbiota by tetracycline was caused by the opportunistic pathogen Serratia sp. increased infections and shortened the life span of bees (BonillaRosso \& Engel, 2018).

Air pollution, microplastics and heavy metals also negatively affect the honeybee gut microbiome composition (Mutlu et al., 2018; Costa et al., 2019; Rothman, Leger, Kirkwood, \& McFrederick, 2019).

\section{Conclusion}

Honey bee microbiota and microbiome are very important for growth, development, metabolism and immune system. It is especially effective in inhibiting pathogens in bee health. Misfeeding, mistakes in colony management and beekeeping practices, unnecessary and continuous antibiotic applications affect the microbiota negatively. Feeding and practices that support and strengthen the microbiota are extremely important for healthy, strong and highly productive beekeeping.

\section{References}

Agus, A., Planchais, J., \& Sokol, H. (2018). Gut microbiota regulation of tryptophan metabolism in health and disease. Cell host \& microbe, 23(6), 716-724.

Alberoni, D., Gaggìa, F., Baffoni, L., \& Di Gioia, D. (2016). Beneficial microorganisms for honey bees: problems and progresses. Applied microbiology and biotechnology, 100(22), 9469-9482.

Alvarez-Sieiro, P., Montalbán-López, M., Mu, D., \& Kuipers, O. P. (2016). Bacteriocins of lactic acid bacteria: extending the family. Applied microbiology and biotechnology, 100(7), 2939-2951.

Anderson, K. E., Sheehan, T. H., Mott, B. M., Maes, P., Snyder, L., Schwan, M. R., Walton, A., Jones, B. M., \& Corby-Harris, V. (2013). Microbial ecology of the hive and pollination landscape: bacterial associates from floral nectar, the alimentary tract and stored food of honey bees (Apis mellifera). PloS one, 8(12), e83125.

Anderson, K. E., Rodrigues, P. A., Mott, B. M., Maes, P., \& Corby-Harris, V. (2016). Ecological succession in the honey bee gut: shift in lactobacillus strain dominance during early adult development. Microbial ecology, 71(4), 1008-1019. 
Anderson, K. E., \& Ricigliano, V. A. (2017). Honey bee gut dysbiosis: a novel context of disease ecology. Current opinion in insect science, 22, 125-132.

Arredondo, D., Castelli, L., Porrini, M. P., Garrido, P. M., Eguaras, M. J., Zunino, P., \& Antúnez, K. (2018). Lactobacillus kunkeei strains decreased the infection by honey bee pathogens Paenibacillus larvae and Nosema ceranae. Beneficial microbes, 9(2), 279-290.

Azambuja, P., Garcia, E. S., \& Ratcliffe, N. A. (2005). Gut microbiota and parasite transmission by insect vectors. Trends in parasitology, 21(12), 568-572.

Bäckhed, F., Ding, H., Wang, T., Hooper, L. V., Koh, G. Y., Nagy, A., Semenkovich, C. F., \& Gordon, J. I. (2004). The gut microbiota as an environmental factor that regulates fat storage. Proceedings of the National Academy of Sciences of the United States of America, 101(44), 15718-15723.

Belkaid, Y., \& Harrison, O. J. (2017). Homeostatic Immunity and the microbiota. Immunity, 46(4), 562-576.

Bleau, N., Bouslama, S., Giovenazzo, P., \& Derome, N. (2020). Dynamics of the honeybee (Apis mellifera) gut microbiota throughout the overwintering period in Canada. Microorganisms, 8(8), 1146.

Bonilla-Rosso, G., \& Engel, P. (2018). Functional roles and metabolic niches in the honey bee gut microbiota. Current opinion in microbiology, 43, 69-76.

Corby-Harris, V., Snyder, L. A., Schwan, M. R., Maes, P., McFrederick, Q. S., \& Anderson, K. E. (2014). Origin and effect of Alpha 2.2 Acetobacteraceae in honey bee larvae and description of Parasaccharibacter apium gen. nov., sp. nov. Applied and environmental microbiology, 80(24), 7460-7472.

Costa, A., Veca, M., Barberis, M., Tosti, A., Notaro, G., Nava, S., Lazzari, M., Agazzi, M., \& Tangorra, F.M. (2019). Heavy metals on honeybees indicate their concentration in the atmosphere a proof of concept. Italian Journal of Animal Science, 18 (1), 309-315.

Daisley, B. A., Trinder, M., McDowell, T. W., Welle, H., Dube, J. S., Ali, S. N., Leong, H. S., Sumarah, M. W., \& Reid, G. (2017). Neonicotinoid-induced pathogen susceptibility is mitigated by Lactobacillus plantarum immune stimulation in a Drosophila melanogaster model. Scientific reports, 7(1), 2703.

Daisley, B.A., Pitek, A.P., Chmiel, J.A., Gibbons, S., Chernyshova, A.M., Al, K.F., Faragalla, K.M., Burton, J.P., Thompson, G.J., \& Reid, G. (2020). Lactobacillus spp. attenuate antibiotic-induced immune and microbiota dysregulation in honey bees. Communations Biology, 3, 534.

Diaz, T., Del-Val, E., Ayala, R., \& Larsen, J. (2019). Alterations in honey bee gut microorganisms caused by Nosema spp. and pest control methods. Pest management science, 75(3), 835-843.

Dong, Z. X., Li, H. Y., Chen, Y. F., Wang, F., Deng, X. Y., Lin, L. B., Zhang, Q. L., Li, J. L., \& Guo, J. (2020). Colonization of the gut microbiota of honey bee (Apis mellifera) workers at different developmental stages. Microbiology Research, 231, 126370.

Egert, M., \& Simmering, R. (2016). The microbiota of the human skin. Advances in experimental medicine and biology, 902, 61-81.

Engel, P., \& Moran, N. A. (2013a). Functional and evolutionary insights into the simple yet specific gut microbiota of the honey bee from metagenomic analysis. Gut microbes, 4(1), 60-65.
Engel, P., \& Moran, N. A. (2013b). The gut microbiota of insects - diversity in structure and function. FEMS microbiology reviews, 37(5), 699-735.

Evans, J. D., Aronstein, K., Chen, Y. P., Hetru, C., Imler, J. L., Jiang, H., Kanost, M., Thompson, G. J., Zou, Z., \& Hultmark, D. (2006). Immune pathways and defence mechanisms in honey bees Apis mellifera. Insect molecular biology, 15(5), 645-656.

Foligne, B., Nutten, S., Grangette, C., Dennin, V., Goudercourt, D., Poiret, S., Dewulf, J., Brassart, D., Mercenier, A., \& Pot, B. (2007). Correlation between in vitro and in vivo immunomodulatory properties of lactic acid bacteria. World journal of gastroenterology, 13(2), 236-243.

Greiner, T., \& Bäckhed, F. (2011). Effects of the gut microbiota on obesity and glucose homeostasis. Trends in endocrinology and metabolism: TEM, 22(4), 117-123.

Harris, J. W., \& Woodring, J. (1992). Effects of stress, age, season, and source colony on levels of octopamine, dopamine and serotonin in the honey bee (Apis mellifera L.) brain. Journal of Insect Physiology, 38 (1), 29-35.

Hilgarth, M., Redwitz, J., Ehrmann, M. A., Vogel, R. F., \& Jakob, F. (2021). Bombella favorum sp. nov. and Bombella mellum sp. nov., two novel species isolated from the honeycombs of Apis mellifera. International journal of systematic and evolutionary microbiology, 71(2), 10.1099/ijsem.0.004633.

Ihle, K. E., Baker, N. A., \& Amdam, G. V. (2014). Insulin-like peptide response to nutritional input in honey bee workers. Journal of insect physiology, 69, 49-55.

Iorizzo, M., Pannella, G., Lombardi, S. J., Ganassi, S., Testa, B., Succi, M., Sorrentino, E., Petrarca, S., De Cristofaro, A., Coppola, R., \& Tremonte, P. (2020a). Inter- and intra-species diversity of lactic acid bacteria in Apis mellifera ligustica Colonies. Microorganisms, 8(10), 1578.

Iorizzo, M., Lombardi, S. J., Ganassi, S., Testa, B., Ianiro, M., Letizia, F., Succi, M., Tremonte, P., Vergalito, F., Cozzolino, A., Sorrentino, E., Coppola, R., Petrarca, S., Mancini, M., \& Cristofaro, A. (2020b). Antagonistic Activity against Ascosphaera apis and Functional Properties of Lactobacillus kunkeei Strains. Antibiotics (Basel, Switzerland), 9(5), 262.

Jandhyala, S. M., Talukdar, R., Subramanyam, C., Vuyyuru, H., Sasikala, M., \& Nageshwar Reddy, D. (2015). Role of the normal gut microbiota. World journal of gastroenterology, 21(29), 8787-8803.

Jing, T. Z., Qi, F.H., \& Wang, Z.Y (2020). Most dominant roles of insect gut bacteria: Digestion. detoxification or essential nutrient provision? Microbiome, 8(1), 38.

Jones, J. C., Fruciano, C., Hildebrand, F., Al Toufalilia, H., Balfour, N. J., Bork, P., Engel, P., Ratnieks, F. L., \& Hughes, W. O. (2017). Gut microbiota composition is associated with environmental landscape in honey bees. Ecology and evolution, 8(1), 441-451.

Kešnerová, L., Moritz, R., \& Engel, P. (2016). Bartonella apis sp. nov., a honey bee gut symbiont of the class Alphaproteobacteria. International journal of systematic and evolutionary microbiology, 66(1), 414421.

Kešnerová, L., Mars, R., Ellegaard, K. M., Troilo, M., Sauer, U., \& Engel, P. (2017). Disentangling metabolic 
functions of bacteria in the honey bee gut. PLOS biology, 15(12), e2003467.

Kešnerová, L., Emery, O., Troilo, M., Liberti, J., Erkosar, B., \& Engel, P. (2020). Gut microbiota structure differs between honeybees in winter and summer. The ISME journal, 14(3), 801-814.

Kishino, S., Takeuchi, M., Park, S. B., Hirata, A., Kitamura, N., Kunisawa, J., Kiyono, H., Iwamoto, R., Isobe, Y., Arita, M., Arai, H., Ueda, K., Shima, J., Takahashi, S., \& Yokozeki, K. (2013). Polyunsaturated fatty acid saturation by gut lactic acid bacteria affecting host lipid composition. Proceedings of the National Academy of Sciences of the United States of America, 110(44), 17808-17813.

Kwong, W. K., \& Moran, N. A. (2016a). Gut microbial communities of social bees. Nature reviews. Microbiology, 14(6), 374-384.

Kwong, W. K., \& Moran, N. A. (2016b). Apibacter adventoris gen. nov., sp. nov., a member of the phylum Bacteroidetes isolated from honey bees. International journal of systematic and evolutionary microbiology, 66(3), 1323-1329.

Kwong, W. K., Mancenido, A. L., \& Moran, N. A. (2017). Immune system stimulation by the native gut microbiota of honey bees. Royal Society open science, 4(2), 170003.

Kwong, W. K., Steele, M. I., \& Moran, N. A. (2018). Genome sequences of Apibacter spp., gut symbionts of Asian honey bees. Genome biology and evolution, 10(4), 1174-1179.

LeBlanc, J. G., Milani, C., de Giori, G. S., Sesma, F., van Sinderen, D., \& Ventura, M. (2013). Bacteria as vitamin suppliers to their host: a gut microbiota perspective. Current opinion in biotechnology, 24(2), 160-168.

Leger, L., \& McFrederick, Q. S. (2020). The gut-brainmicrobiome axis in bumble bees. Insects, 11(8), 517.

Li, J. H., Evans, J. D., Li, W. F., Zhao, Y. Z., DeGrandi-Hoffman, G., Huang, S. K., Li, Z. G., Hamilton, M., \& Chen, Y. P. (2017). New evidence showing that the destruction of gut bacteria by antibiotic treatment could increase the honey bee's vulnerability to Nosema infection. PloS one, 12(11), e0187505.

Liberti, J., \& Engel, P. (2020). The gut microbiota - brain axis of insects. Current opinion in insect science, 39, 6-13.

Maragkoudakis, P. A., Chingwaru, W., Gradisnik, L., Tsakalidou, E., \& Cencic, A. (2010). Lactic acid bacteria efficiently protect human and animal intestinal epithelial and immune cells from enteric virus infection. International journal of food microbiology, 141 Suppl 1, S91-S97.

Martinson, V. G., Danforth, B. N., Minckley, R. L., Rueppel, O., Tingek, S., \& Moran, N. A. (2011). A simple and distinctive microbiota associated with honey bees and bumble bees. Molecular Ecology, 20 (3), 619-628.

Martinson, V. G., Moy, J., \& Moran, N. A. (2012). Establishment of characteristic gut bacteria during development of the honeybee worker. Applied and environmental microbiology, 78(8), 2830-2840.

Milani, C., Duranti, S., Mangifesta, M., Lugli, G. A., Turroni, F., Mancabelli, L., Viappiani, A., Anzalone, R., Alessandri, G., Ossiprandi, M. C., van Sinderen, D., \& Ventura, M. (2018). Phylotype-level profiling of lactobacilli in highly complex environments by means of an internal transcribed spacer-based metagenomic approach. Applied and microbiology, 84(14), e00706-18.

Mollet, J. C., Leroux, C., Dardelle, F., \& Lehner, A. (2013). Cell wall composition, biosynthesis and remodeling during pollen tube growth. Plants (Basel, Switzerland), 2(1), 107-147.

Molloy, M. J., Bouladoux, N., \& Belkaid, Y. (2012). Intestinal microbiota: shaping local and systemic immune responses. Seminars in immunology, 24(1), 58-66.

Moran, N. A., Hansen, A. K., Powell, J. E., \& Sabree, Z. L. (2012). Distinctive gut microbiota of honey bees assessed using deep sampling from individual worker bees. PloS one, 7(4), e36393.

Motta, E., Raymann, K., \& Moran, N. A. (2018). Glyphosate perturbs the gut microbiota of honey bees. Proceedings of the National Academy of Sciences of the United States of America, 115(41), 10305-10310.

Mutlu, E. A., Comba, I. Y., Cho, T., Engen, P. A., Yazıcı, C., Soberanes, S., Hamanaka, R. B., Niğdelioğlu, R., Meliton, A. Y., Ghio, A. J., Budinger, G., \& Mutlu, G. M. (2018). Inhalational exposure to particulate matter air pollution alters the composition of the gut microbiome. Environmental pollution (Barking, Essex: 1987), 240, 817-830.

Nicholson, J. K., Holmes, E., Kinross, J., Burcelin, R., Gibson, G., Jia, W., \& Pettersson, S. (2012). Host-gut microbiota metabolic interactions. Science (New York, N.Y.), 336(6086), 1262-1267.

Olofsson, T. C., Alsterfjord, M., Nilson, B., Butler, È., \& Vásquez, A. (2014). Lactobacillus apinorum sp. nov., Lactobacillus mellifer sp. nov., Lactobacillus mellis sp. nov., Lactobacillus melliventris sp. nov., Lactobacillus kimbladii sp. nov., Lactobacillus helsingborgensis sp. nov. and Lactobacillus kullabergensis sp. nov., isolated from the honey stomach of the honeybee Apis mellifera. International journal of systematic and evolutionary microbiology, 64(Pt 9), 3109-3119.

Pachila, A., Ptaszynska, A.A., Wicha, M., Olenska, E., \& Małek, W. (2017). Fascinating fructophilic lactic acid bacteria associated with various fructose-rich niches. Annales Universitatis Mariae Curie Sklodowska Med, 72, S41S50.

Pascale, A., Marchesi, N., Marelli, C., Coppola, A., Luzi, L., Govoni, S., Giustina, A., Gagliardi, A., Totino, V., Cacciotti, F., lebba, V., Neroni, B., Bonfiglio, G., Trancassini, M., Passariello, C., Pantanella, F., \& Schippa, S. (2018). Rebuilding the gut microbiota ecosystem. International journal of environmental research and public health, 15(8), 1679.

Peghaire, E., Moné, A., Delbac, F., Debroas, D., ChaucheyrasDurand, F., \& El Alaoui, H. (2020). A Pediococcus strain to rescue honeybees by decreasing Nosema ceranaeand pesticide-induced adverse effects. Pesticide biochemistry and physiology, 163, 138-146.

Pernice, M., Simpson, S. J., \& Ponton, F. (2014). Towards an integrated understanding of gut microbiota using insects as model systems. Journal of insect physiology, 69, 12-18.

Piccart, K., Vásquez, A., Piepers, S., De Vliegher, S., \& Olofsson, T. C. (2016). Short communication: Lactic acid bacteria from the honeybee inhibit the in vitro growth of mastitis pathogens. Journal of dairy science, 99(4), 2940-2944.

Powell, J. E., Martinson, V. G., Urban-Mead, K., \& Moran, N. A. (2014). Routes of Acquisition of the gut microbiota 
of the honey bee Apis mellifera. Applied and environmental microbiology, 80(23), 7378-7387.

Purchiaroni, F., Tortora, A., Gabrielli, M., Bertucci, F., Gigante, G., Ianiro, G., Ojetti, V., Scarpellini, E., \& Gasbarrini, A. (2013). The role of intestinal microbiota and the immune system. European review for medical and pharmacological sciences, 17(3), 323-333.

Raymann, K., Motta, E., Girard, C., Riddington, I. M., Dinser, J. A., \& Moran, N. A. (2018a). Imidacloprid decreases honey bee survival rates but does not affect the gut microbiome. Applied and environmental microbiology, 84(13), e00545-18.

Raymann, K., Bobay, L. M., \& Moran, N. A. (2018b). Antibiotics reduce genetic diversity of core species in the honeybee gut microbiome. Molecular ecology, 27(8), 2057-2066.

Rothman, J. A., Leger, L., Kirkwood, J. S., \& McFrederick, Q. S. (2019). Cadmium and selenate exposure affects the honey bee microbiome and metabolome, and beeassociated bacteria show potential for bioaccumulation. Applied and environmental microbiology, 85(21), e01411-19.

Rouzé, R., Moné, A., Delbac, F., Belzunces, L., \& Blot, N. (2019). The honeybee gut microbiota is altered after chronic exposure to different families of Insecticides and infection by Nosema ceranae. Microbes and environments, 34(3), 226-233.

Schroeder, B. O., \& Bäckhed, F. (2016). Signals from the gut microbiota to distant organs in physiology and disease. Nature medicine, 22(10), 1079-1089.

Sekirov, I., Russell, S. L., Antunes, L. C., \& Finlay, B. B. (2010). Gut microbiota in health and disease. Physiological reviews, 90(3), 859-904.

Serna-Cock, L., Rojas-Dorado, M., Ordoñez-Artunduaga, D., García-Salazar, A., García-González, E., \& Aguilar, C. N. (2019). Crude extracts of metabolites from co-cultures of lactic acid bacteria are highly antagonists of Listeria monocytogenes. Heliyon, 5(9), e02448.

Shi,W., Syrenne, R., Sun, J.-Z., \& Yuan, J.S. (2010). Molecular approaches to study the insect gut symbiotic microbiota at the "omics" age. Insect Science, 17, 199219.

Silva, M.S., Rabadzhiev, Y., Renon Eller, M., Iliev, I., Ivanova, I., \& Santana, W.C. (2017). Microorganisms in honey. Honey analysis, S233-S258.

Sommer, F., \& Bäckhed, F. (2013). The gut microbiota-masters of host development and physiology. Nature reviews. Microbiology, 11(4), 227-238.

Subotic, S., Boddicker, A. M., Nguyen, V. M., Rivers, J., Briles, C. E., \& Mosier, A. C. (2019). Honey bee microbiome associated with different hive and sample types over a honey production season. PloS one, 14(11), e0223834.

Tola, Y. H., Waweru, J. W., Hurst, G., Slippers, B., \& Paredes, J. C. (2020). Characterization of the Kenyan honey bee (Apis mellifera) gut microbiota: a first look at tropical and sub-saharan African bee associated microbiomes. Microorganisms, 8(11), 1721.
Valentini, M., Piermattei, A., Di Sante, G., Migliara, G., Delogu, G., \& Ria, F. (2014). Immunomodulation by gut microbiota: role of toll-like receptor expressed by $\mathrm{t}$ cells. Journal of immunology research, 2014, 586939.

Vásquez, A., Forsgren, E., Fries, I., Paxton, R. J., Flaberg, E., Szekely, L., \& Olofsson, T. C. (2012). Symbionts as major modulators of insect health: lactic acid bacteria and honeybees. PloS one, 7(3), e33188.

Vergnolle, N. (2016). Protease inhibition as new therapeutic strategy for GI diseases. Gut, 65(7), 1215-1224.

Vernier, C.L., Chin, I.M., Adu-Oppong, B., Krupp, J.J., Levine, J., Dantas, G., \& Ben-Shahar, Y. (2020). The gut microbiome defines social group membership in honey bee colonies. Sciences Advances, 6 (42), eabd3431.

Wang, H., Liu, C., Liu, Z., Wang, Y., Ma, L., \& Xu, B. (2020).The different dietary sugars modulate the composition of the gut microbiota in honeybee during overwintering. BMC Microbiology, 20 (1), 61.

Wang, X., Zhong, Z., Chen, X., Hong, Z., Lin, W., Mu, X., Hu, X., \& Zheng, H. (2021). High-fat diets with differential fatty acids Induce obesity and perturb gut microbiota in honey bee. International journal of molecular sciences, 22(2), 834.

Westfall, S., Lomis, N., \& Prakash, S. (2018). Longevity extension in Drosophila through gut-brain communication. Scientific Reports, 8, 8362.

Wu, M., Sugimura, Y., Iwata, K., Takaya, N., Takamatsu, D., Kobayashi, M., Taylor, D., Kimura, K., \& Yoshiyama, M. (2014). Inhibitory effect of gut bacteria from the Japanese honey bee, Apis cerana japonica, against Melissococcus plutonius, the causal agent of European foulbrood disease. Journal of insect science (Online), 14, 129.

Wu, Y., Zheng, Y., Chen, Y., Wang, S., Chen, Y., Hu, F., \& Zheng, H. (2020). Honey bee (Apis mellifera) gut microbiota promotes host endogenous detoxification capability via regulation of $\mathrm{P} 450$ gene expression in the digestive tract. Microbial Biotechnology, 13(4), 12011212.

Yiu, J. H., Dorweiler, B., \& Woo, C. W. (2017). Interaction between gut microbiota and toll-like receptor: from immunity to metabolism. Journal of molecular medicine (Berlin, Germany), 95(1), 13-20.

Zheng, H., Powell, J. E., Steele, M. I., Dietrich, C., \& Moran, N. A. (2017). Honeybee gut microbiota promotes host weight gain via bacterial metabolism and hormonal signaling. Proceedings of the National Academy of Sciences of the United States of America, 114(18), 4775-4780.

Zheng, H., Steele, M. I., Leonard, S. P., Motta, E., \& Moran, N. A. (2018). Honey bees as models for gut microbiota research. Lab animal, 47(11), 317-325.

Zhu, L., Qi, S., Xue, X., Niu, X., \& Wu, L. (2020). Nitenpyram disturbs gut microbiota and influences metabolic homeostasis and immunity in honey bee (Apis mellifera L.). Environmental pollution (Barking, Essex: 1987), 258, 113671. 